\title{
Detection of Magnetic Contaminations in Industrial Products Using HTS SQUIDs
}

\author{
Hans-Joachim Krause, Grigory I. Panaitov, Norbert Wolters, Dieter Lomparski, Willi Zander, Yi Zhang, \\ Elmar Oberdoerffer, Dirk Wollersheim, and Winfried Wilke
}

\begin{abstract}
Many products in the pharmaceutical and food industry are packaged in metallized wrappings. With standard high-frequency search coil metal detectors, they can only be tested for metal contaminations before they are wrapped. However, a key requirement of industrial quality control is the inspection of the products at the end of the production line. We have developed an inspection system for detecting the magnetic remanence of the contaminants. The system utilizes two HTS rf SQUID magnetometers with step edge junctions immersed in liquid nitrogen. The SQUIDs are arranged such that they cover the product channel in a rotated planar electronic gradiometer configuration. In order to suppress the low-frequency magnetic disturbances typically found in industrial environment, the product channel and the SQUID system were mounted inside a coaxial three-layer Mumetal shield. In combination with the gradiometric suppression, homogeneous low-frequency disturbance fields were attenuated by a factor of 400,000 . The sensitivity of the system for small magnetic particles was determined experimentally, using numerous steel balls and splinters. A stainless steel particle of $3 \mu \mathrm{g}$, corresponding to a sphere diameter of $0.09 \mathrm{~mm}$, was detected with and without aluminized wrapping.
\end{abstract}

Index Terms-Metal detector, nondestructive testing, remote magnetometry, SQUIDs.

\section{INTRODUCTION}

$\mathbf{P}$ RODUCTS in the pharmaceutical and food industry are tested for metal inclusions which may contaminate the products due to machine wear in industrial production lines. Swarfs, splinters or chips may break off the machines and may mix with the products being manufactured or processed. State of the art in industrial metal detection are search coil detectors on the basis of high-frequency eddy current detection. Metallic contaminants of sub-mm size may be safely detected, provided that the products are nonconducting.

In many cases, the products can only be inspected before they are wrapped and packaged because the wrappings are metallized with an aluminum coating of a few micrometers thickness. The wrapping gives such a strong eddy current signal that the sensitivity of the search coil detector has to be reduced below acceptable levels. Hence, it is generally not possible with search

Manuscript received October 5, 2004. This work was supported by the German BMBF under Grants no. 13N7738 and 13N7739.

H.-J. Krause, G. I. Panaitov, N. Wolters, D. Lomparski, W. Zander, and Y. Zhang are with Forschungszentrum Jülich, Juelich 52425, Germany (e-mail: h.-j.krause@fz-juelich.de).

E. Oberdoerffer is with Sartorius Aachen GmbH\&Co. KG (formerly Boekels), Aachen 52070, Germany.

D. Wollersheim was with Boekels and is now with Ortungstechnik Ing. K. Ebinger, Köln 51149, Germany.

W. Wilke was with Boekels and is now with the University of Applied Sciences Würzburg-Schweinfurt, Schweinfurt 97421, Germany.

Digital Object Identifier 10.1109/TASC.2005.850027 coil metal detectors to find metal inclusions through aluminized wrappings. However, a key requirement of industrial quality control is the inspection of the products at the end of the production line.

The most commonly used material in production machines is stainless steel. Contamination and wear from these machines may be magnetized and their static magnetic remanence field may be detected. Remote magnetometry [1] is advantageous for magnetic particle detection in nonmagnetic products since the measurement is not impaired by electrically conducting products or wrappings. The use of SQUID magnetometers allows the realization of excellent sensitivities. The applicability of HTS SQUID systems has been experimentally demonstrated for detecting of fine iron particles in wires [2], for localizing ferrous inclusions in aircraft gas turbine discs [3] and for finding metallic contaminants in food [4]-[6].

\section{System Design AND SETUP}

The development work included calculation of the optimum SQUID sensor configuration, design of a Mumetal shielding for attenuation of low-frequency magnetic disturbances and a rf shield for the sensors, a two-channel readout electronics for the SQUIDs including a summation unit with filters (highpass, $50 \mathrm{~Hz}$ notch and 8th order lowpass), all remotely adjustable by a control and data acquisition software. In order to satisfy the envisioned industrial application requirements, the product channel cross section (functional orifice) was defined to measure $80 \mathrm{~mm} \times 25 \mathrm{~mm}$. Using the magnetic dipole law,

$$
B=\frac{\mu_{0}}{4 \pi}\left(\frac{3 \mathbf{m} \cdot \mathbf{r}}{r^{5}} \mathbf{r}-\frac{\mathbf{m}}{r^{3}}\right)
$$

possible signal traces of $\mathrm{x}-, \mathrm{y}$ - and $\mathrm{z}$ - oriented dipoles moving at constant speed in $\mathrm{x}$-direction were calculated for different two-and three-SQUID arrangements. The so-called rotated planar electronic gradiometer configuration depicted in Fig. 1 (left) was found to be the optimum arrangement for two SQUIDs. The results of the calculated signal traces are displayed in Fig. 2.

A particle is called 'detectable' if the peak-to-peak SQUID gradiometer signal exceeds the noise level of the system in 30 $\mathrm{Hz}$ bandwidth $(\sim 3 \mathrm{pT})$ by a safety factor of 4 . By performing a scaling of the calculated sensitivity map, graphs of the safely detectable magnetic moments for all three orthogonal magnetization directions were derived, see. Fig. 3. Whereas in the most favorable case of a z-oriented magnetic particle passing directly underneath one magnetometer, a moment as low as 6 . 


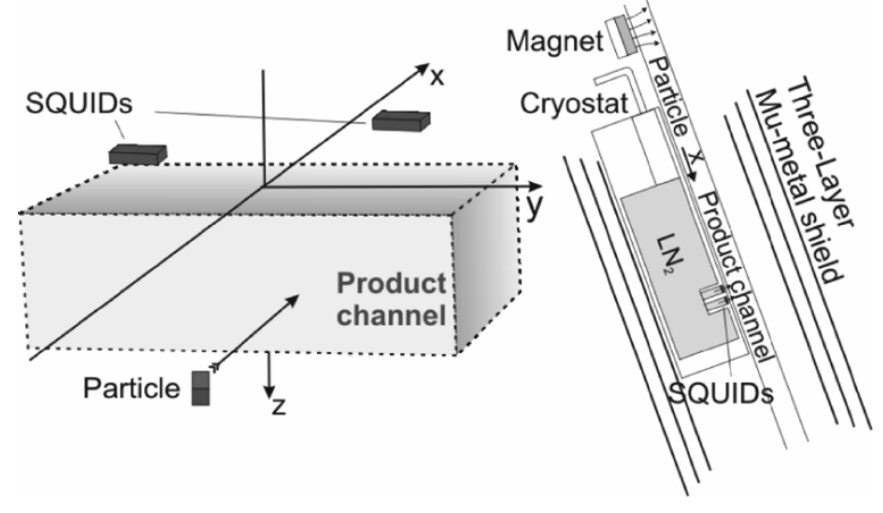

Fig. 1. Left: Principle of particle detection. Two HTS SQUID magnetometers are arranged above the product channel where the pre-magnetized industrial products are passing through during inspection. Right: Schematic of the system setup with cryostat and product slide inside coaxial Mumetal shield. At system entry, the products are exposed to a magnetizing field of $60 \mathrm{mT}$.

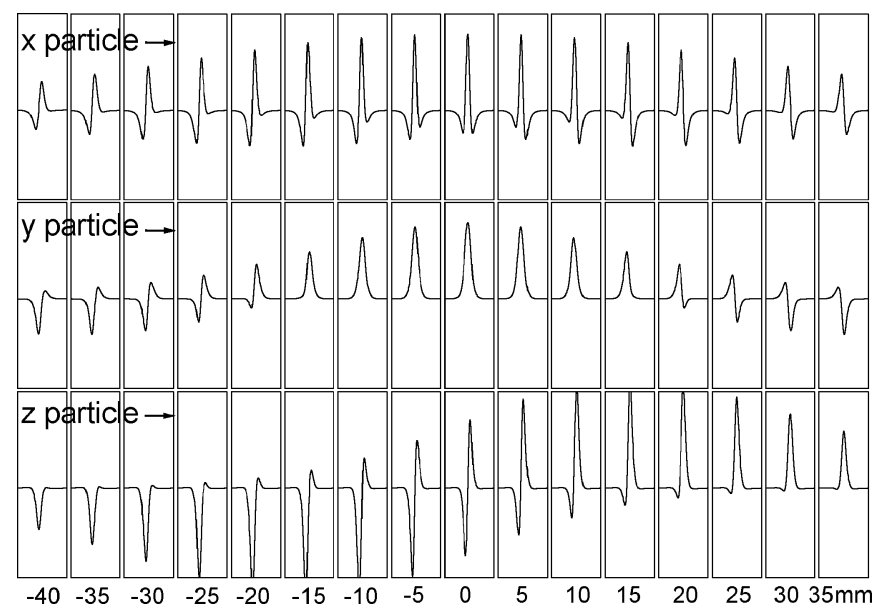

Fig. 2. Simulated signals of magnetic particles passing two SQUID magnetometers in the rotated planar gradiometer configuration depicted in Fig. 1. The gradiometer baseline is $34 \mathrm{~mm}$, the rotation angle $45^{\circ}$. The coordinates of the SQUID positions are $\mathrm{x}=-12 \mathrm{~mm}, \mathrm{y}=-12 \mathrm{~mm}$, $\mathrm{z}=-18 \mathrm{~mm}$ and $\mathrm{x}=12 \mathrm{~mm}, \mathrm{y}=12 \mathrm{~mm}, \mathrm{z}=-18 \mathrm{~mm}$, respectively. A magnetic particle with a magnetization of $1.9 \times 10^{-5} \mathrm{Am}^{2}$ in $\mathrm{x}$-direction (top row), y-direction (middle row) or z-direction (bottom row), moving at constant speed in $\mathrm{x}$-direction, passes the gradiometer at $\mathrm{z}=25 \mathrm{~mm}$, and at $\mathrm{y}$ varying from -40 to $+35 \mathrm{~mm}$. The horizontal spatial scale of each signal graph is $420 \mathrm{~mm}$, the vertical magnetic field scale is $70 \mathrm{nT}$.

$10^{-11} \mathrm{Am}^{2}$ may be safely detected, the limit of safe detection at an arbitrary position in the functional orifice is $6 \cdot 10^{-9} \mathrm{Am}^{2}$.

As SQUID sensors, HTS rf SQUID magnetometers with $3.5 \mathrm{~mm}$ YBCO washers, $100 \mu \mathrm{m} \times 100 \mu \mathrm{m}$ loop and step-edge junctions were used in conjunction with $10 \mathrm{~mm} \times 10 \mathrm{~mm}$ $\mathrm{SrTiO}_{3}$ substrate resonators (effective area $0.65 \mathrm{~mm}^{2}$ ) [7].

In order to attain a robust industry-grade setup satisfying the strict rules of pharmacological and food industry, a cryostat made of stainless steel was manufactured (Juelicher SQUID $\mathrm{GmbH})$. It was laid out for distance minimization between SQUID sensors and product channel. For this purpose, the SQUID magnetometers were mounted such that they were immersed in liquid nitrogen in cylindrical stainless steel containers with a minimum vacuum distance of $1 \mathrm{~mm}$ to a stainless steel lid. The cryostat with this SQUID window was rigidly fixed underneath the product channel within the three-layer

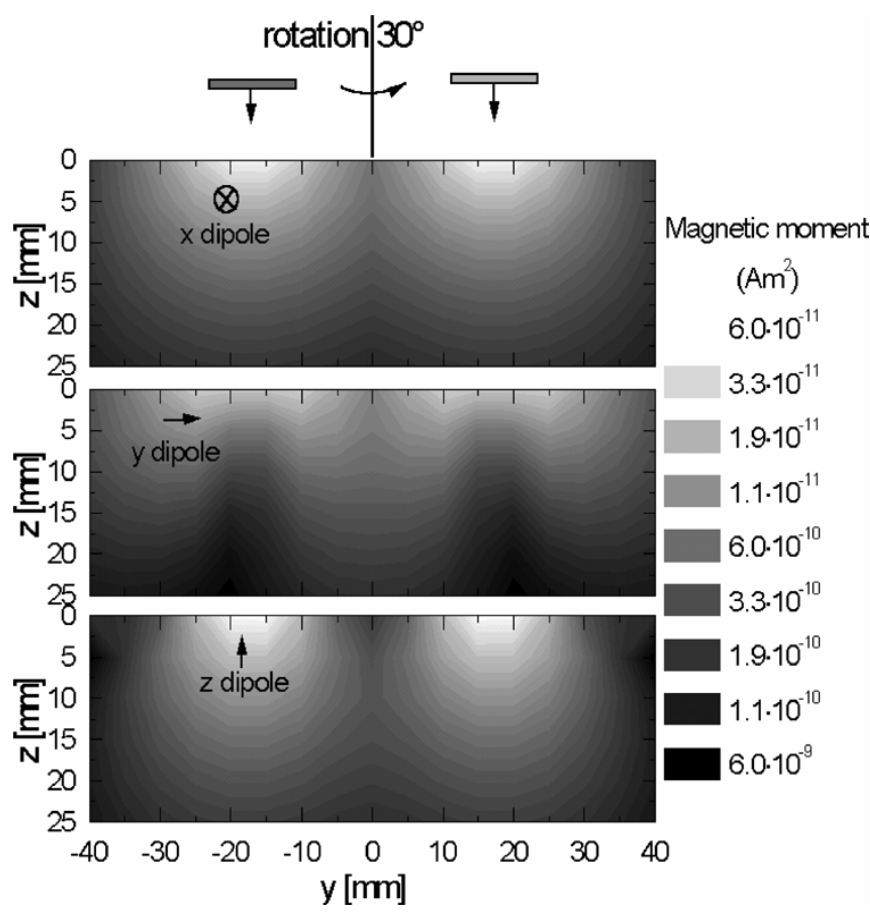

Fig. 3. Simulated sensitivity in the measurement channel for a rotated planar gradiometer as depicted in Fig. 1. In the calculation, a rotation angle of $30^{\circ}$ proved superior over $45^{\circ}$. The SQUID sensor locations are $\mathrm{x}=-10 \mathrm{~mm}, \mathrm{y}=$ $-17.3 \mathrm{~mm}, \mathrm{z}=-10 \mathrm{~mm}$ and $\mathrm{x}=10 \mathrm{~mm}, \mathrm{y}=17.3 \mathrm{~mm}, \mathrm{z}=-10 \mathrm{~mm}$, respectively. Plotted is the minimum detectable magnetic moment of a particle passing the product channel in $\mathrm{x}$ direction for the magnetization orientations $\mathrm{x}$ (top), $\mathrm{y}$ (middle) and $\mathrm{z}$ (bottom graph) as a function of the position $\mathrm{y}$ and $\mathrm{z}$ where the particle passes the channel. The magnetic moment was determined by calculating the maximum signal swing for a given moment and by relating the calculated peak-to-peak amplitude with the required $12 \mathrm{pT}$ field variation.

Mumetal shield. The whole system arrangement was tilted by about $70^{\circ}$ with respect to the horizontal in order to guarantee reliable sliding of the products (tablet or food boxes) past the SQUID sensors (see Fig. 1, right).

In an unshielded industrial environment, one typically finds strong low-frequency magnetic disturbances. In addition to power-line related AC noise, signals originating from moving magnetic objects such as fork-lifts, machinery and tools within the production hall, and cars and trucks in the vicinity have to be suppressed. The typical SQUID gradiometer balance of about 1000 does not suffice. In order to make use of the SQUID sensitivity in this magnetically disturbed environment, the system has to be equipped with an additional passive shielding enclosing the product channel and the SQUID gradiometer. A cylindrical shielding consisting of up to three coaxial tubes (diameter $\varnothing=180 \mathrm{~mm}$, length $l=720 \mathrm{~mm} ; \varnothing=220 \mathrm{~mm}$, $l=760 \mathrm{~mm} ; \varnothing=260 \mathrm{~mm}, l=800 \mathrm{~mm}$ ) was manufactured from $1.5 \mathrm{~mm}$ thick Mumetal plates (Vacuumschmelze, Hanau, Germany).

\section{Characterization OF THE SYSTEM}

The attenuation factors of the coaxial Mumetal shield for homogeneous static magnetic fields were measured inside a $2 \mathrm{~m}$ $\times 2 \mathrm{~m}$ Helmholtz coil. The values are listed in Table I for 1, 2 , and 3 Mumetal layers. The values measured in the radial direction are relevant because the gradiometer sensors measure in 
TABLE I

MEASURED SHIELding FACTORS OF CYLINDRICAL MuMETAL SHIELD

\begin{tabular}{ccccc}
\hline \hline Direction & & \multicolumn{2}{c}{ Number of coaxial shielding tubes } \\
\hline Field & Measurement & 1 & 2 & 3 \\
\hline radial & radial & 20,000 & 47,500 & 49,300 \\
axial & radial & 6,700 & 19,000 & 74,000 \\
axial & axial & 345 & 1,730 & 2,180 \\
radial & axial & 13,100 & 4,500 & 61,700 \\
\hline \hline
\end{tabular}

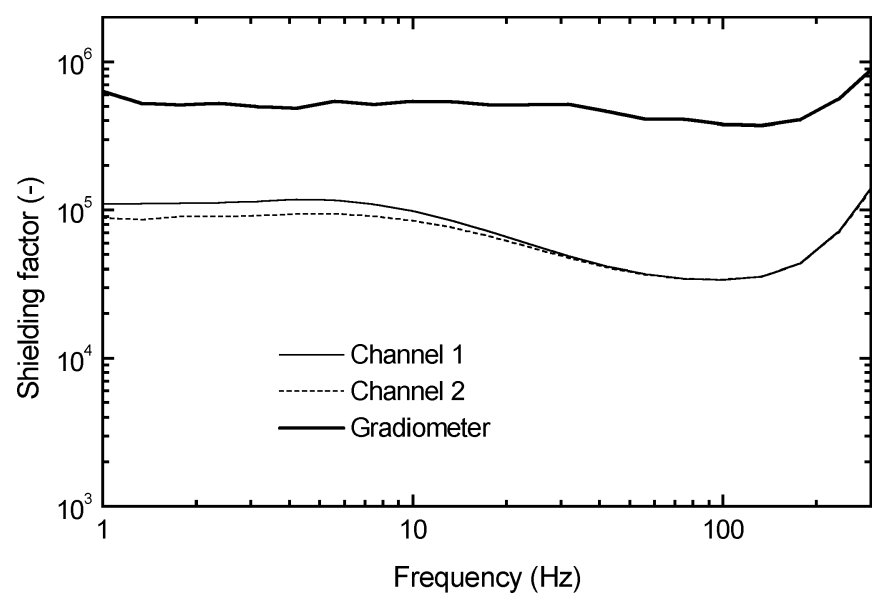

Fig. 4. Measured attenuation of homogeneous radial magnetic fields as a function of frequency for the system in a coaxial 3-layer Mumetal shielding. The thin solid and the dotted line represent the passive shielding factor of the Mumetal tubes, the thick solid line denotes the total attenuation factor.

that direction when the stainless steel dewar is mounted in the three-layer shield. The combination of active gradiometric and passive Mumetal disturbance suppression led to a total attenuation factor of 400000 for homogeneous low-frequency disturbance fields (cf. Fig. 4). The attainable attenuation is limited by phase shifts in the steel: with a fiberglass dewar, a shielding factor of 2000000 was found.

Fig. 5 displays the flux noise of the SQUID magnetometers measured before and after mounting them in the stainless steel cryostat. It is noted that a slight increase of noise $(\sim 20 \%)$ is found due to the thermal noise contribution of the stainless steel walls of the cryostat. By replacing the stainless steel lid and SQUID containers with titanium alloy components, this additional noise could be reduced.

In order to have a feeling for the size of magnetic disturbance signals, the magnetic moments of numerous magnetic objects were determined by scanning them past a triaxial fluxgate magnetometer. By fitting the measured traces with the dipole law (1), the magnetic moments were obtained. For illustration, a few examples are given: Screwdriver: $11.3 \mathrm{Am}^{2}$, manual transport cart (trolley): $4.3 \mathrm{Am}^{2}$, fork lift: $8.9 \mathrm{Am}^{2}$, passenger car Seat Marbella: $122 \mathrm{Am}^{2}$, small truck Daimler Benz 208: $1450 \mathrm{Am}^{2}$. Note that the variability of moments is enormous. Therefore, we tried to compile a classification of disturbers in order to define circles around the system where magnetic objects have to stay out, see Table II. This means that tools and transport trolleys have to stay away at least $94 \mathrm{~cm}$ from our system. Without the Mumetal shielding, their 'no-go area' would be as large as $6.93 \mathrm{~m}$.

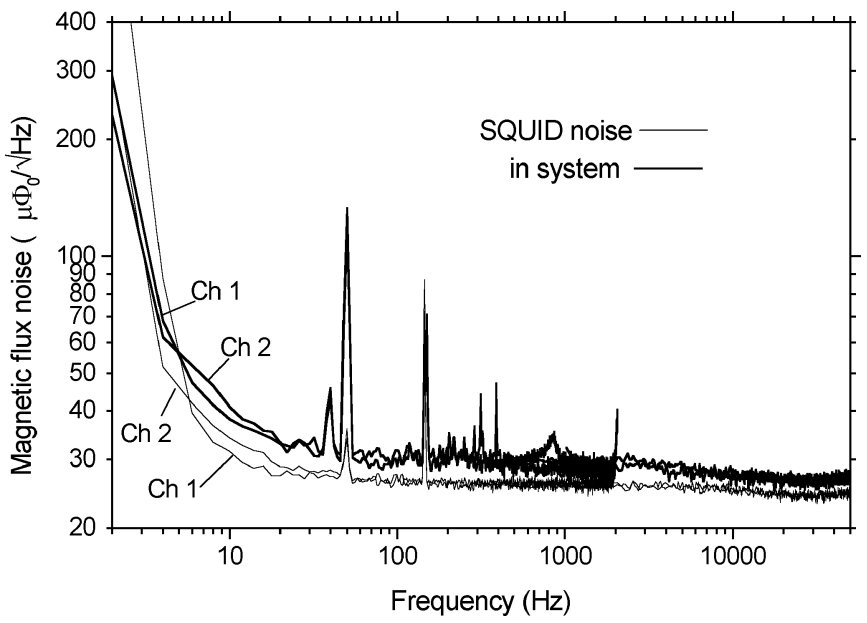

Fig. 5. Measured flux noise spectra of the SQUID sensors before and after mounting in the stainless steel dewar. A slight enhancement of the noise due to the thermal noise of the stainless steel window close to the sensors was observed.

TABLE II

ObJect Classes for Magnetic Disturbers AND REQUIRED MINIMUM DISTANCE FROM THE SQUID SYSTEM

\begin{tabular}{lccc}
\hline \hline \multicolumn{1}{c}{ Shielding factor of SQUID system: } & 1,000 & 400,000 \\
\hline Object class & Magnetic moment & Required minimum distance \\
\hline Ironmongery & $2 \mathrm{Am}^{2}$ & $3.22 \mathrm{~m}$ & $0.44 \mathrm{~m}$ \\
Tools \& Transport & $20 \mathrm{Am}^{2}$ & $6.93 \mathrm{~m}$ & $0.94 \mathrm{~m}$ \\
Passenger cars & $200 \mathrm{Am}^{2}$ & $14.9 \mathrm{~m}$ & $2.03 \mathrm{~m}$ \\
Trucks & $2000 \mathrm{Am}^{2}$ & $32.2 \mathrm{~m}$ & $4.37 \mathrm{~m}$ \\
\hline \hline
\end{tabular}

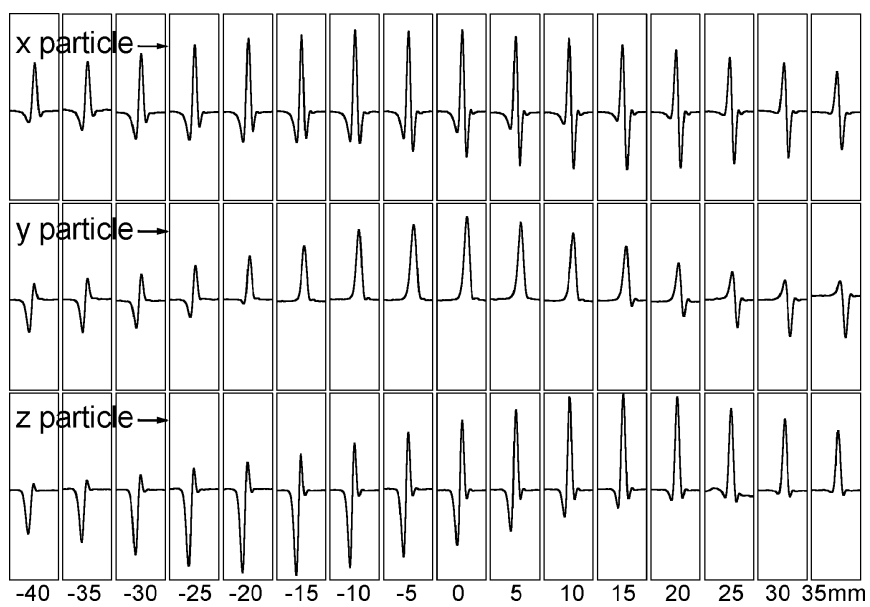

Fig. 6. Measured signals of a magnetic particle (magnetization oriented in $\mathrm{x}$ $\mathrm{y}$ - and $\mathrm{z}$-direction) passing the rotated planar SQUID gradiometer at positions $\mathrm{y}$ varying from -40 to $+35 \mathrm{~mm}$. The coordinates of the SQUID positions are the same as in the simulations of Fig. 2. The box around each graph has horizontal scale of $0.5 \mathrm{~s}$ and a vertical scale of $70 \mathrm{nT}$.

\section{Measurement of Magnetic Test Particles}

Fig. 6 shows measured signals of a magnetic particle passing the product channel at different lateral positions $y$.

The experimental signal traces are in excellent agreement with the simulated traces of Fig. 2. The measurement depicted in Fig. 7 demonstrates that metallized product wrappings have negligible influence. The signal trace of a stainless steel particle 


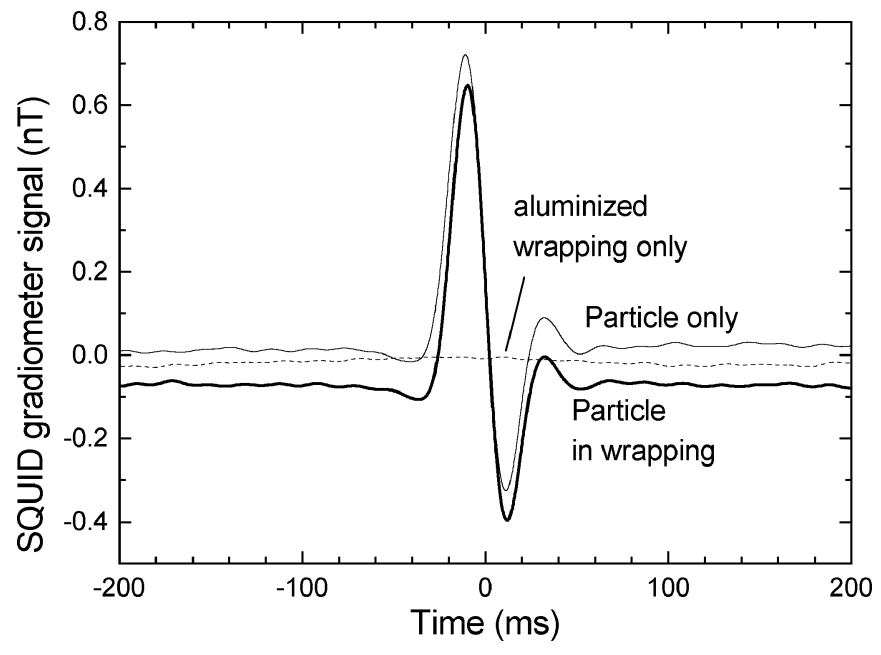

Fig. 7. SQUID gradiometer signal of a metal particle (stainless steel ball, VA 1.4034 , diameter $0.8 \mathrm{~mm}$, magnetic moment $8 \times 10^{-7} \mathrm{Am}^{2}$ ), passing the sensor plane at a distance of about $50 \mathrm{~mm}$. The thin solid line shows the trace measured with the particle alone, the thick solid line was obtained when the particle was put inside an aluminum-coated wrapping. For comparison, the dotted line represents the signal of the wrapping alone.

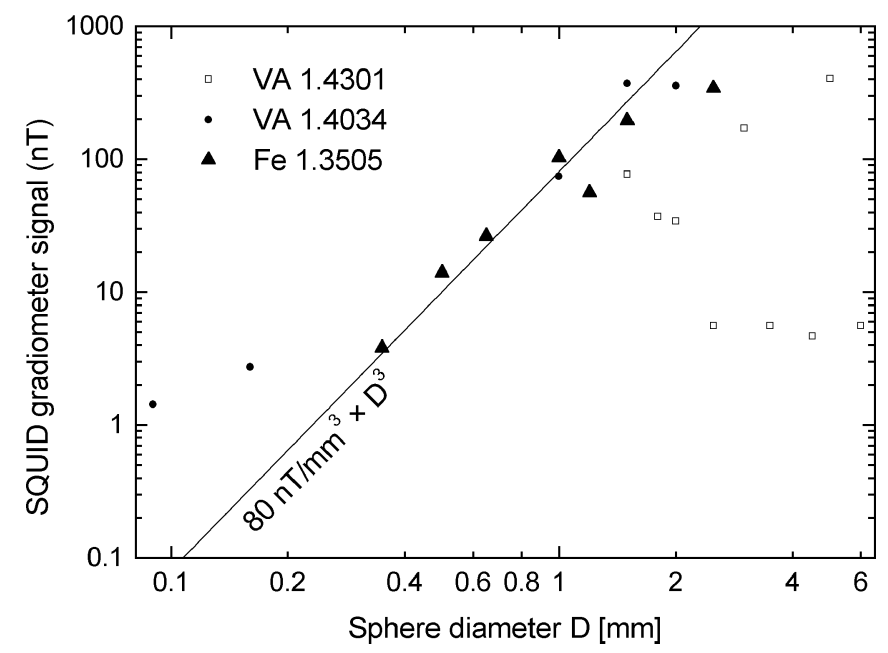

Fig. 8. Measured peak signals of different particles as a function of their diameter. The particles passed the SQUID gradiometer plane at a distance $\mathbf{r}_{0}$ of about $20 \mathrm{~mm}$. For small Fe 1.3505 balls, the signals scale well with the particle volume, as calculated using Eq. (2) (straight line).

measured with and without aluminum-coated package is almost identical. The same holds true for tablet blister packs.

Systematic experiments were conducted with numerous magnetic test objects. Steel balls of three different material grades were examined: stainless steels VA 1.4034 (X46Cr13, AISI 420) and VA 1.4301 (X5CrNi1810, AISI 304) and chrome steel Fe 1.3505 (100Cr6, AISI 52 100). In Fig. 8, the maximum signal swing is plotted versus the diameter of the balls, ranging from $0.35 \mathrm{~mm}$ to $6 \mathrm{~mm}$. Two splinters from a VA 1.4034 sieve, with a mass of $3 \mu \mathrm{g}$ and $17 \mu \mathrm{g}$, respectively, were also measured. Their signals are displayed in Fig. 8 vs. their equivalent sphere diameters of $0.09 \mathrm{~mm}$ and $0.16 \mathrm{~mm}$.
As expected, no systematic size dependence was observed for stainless steel balls, since their magnetism depends on the martensitic phase content which is affected by the mechanical history of the balls. The signals of the small chrome steel spheres, however, scaled well with the volume. The magnetization of a sphere is given by $\mathrm{M}=3 \mu_{\mathrm{r}} \mathrm{H}_{\mathrm{c}} /\left(2+\mu_{\mathrm{r}}\right)$. Approximating the relative permeability with a linear relation in the second quadrant, $\mu_{\mathrm{r}}=\mathrm{B}_{\mathrm{r}} /\left(\mu_{0} \mathrm{H}_{\mathrm{c}}\right)$, and adopting typical values for the remanence, $\mathrm{B}_{\mathrm{r}}=0.5 \mathrm{~T}$, and the coercivity, $\mathrm{H}_{\mathrm{c}}=2000 \mathrm{~A} / \mathrm{m}, \mathrm{M}=6000 \mathrm{~A} / \mathrm{m}$ is obtained. The axial magnetic field $B_{z}$ at a distance $r_{0}=20 \mathrm{~mm}$ is then given by

$$
B_{z}=\frac{\mu_{0} m_{z}}{2 \pi r_{0}^{3}}=\frac{\mu_{0} D^{3} M}{12 r_{0}^{3}} \approx 80 \frac{\mathrm{nT}}{\mathrm{mm}^{3}} \cdot D^{3} \text {. }
$$

as graphically displayed by the solid line in Fig. 8 .

\section{CONCLUSION}

A testing technique based on recording the remanent magnetic field of particles using HTS SQUID sensors was developed. The system is comprised of two SQUID magnetometers covering the product channel in a rotated planar gradiometer configuration, mounted in a stainless steel cryostat and enclosed by a three-layer coaxial Mumetal shield. The combination of active gradiometric and passive Mumetal disturbance suppression led to an attenuation factor of 400000 for homogeneous low-frequency disturbance fields.

The sensitivity of the SQUID system for very small magnetic particles was experimentally determined using spheres of different materials and diameters. For steel balls, a good agreement with theoretically predicted values were found. Stainless steel wires with particle masses corresponding to sphere diameters of down to $0.09 \mathrm{~mm}$ were found to be detectable. In contrast to conventional search coil metal detectors, the sensitivity is not impaired by wrappings with metallization such as tablet blister packs. Thus, the SQUID system was found superior for the inspection of wrapped products.

\section{REFERENCES}

[1] G. B. Donaldson, A. Cochran, and D. M. McKirdy, "The use of SQUIDs for NDE," in SQUID Sensors, H. Weinstock, Ed. Dordrecht: Kluwer, 1996, pp. 599-628.

[2] H. Itozaki, T. Nagaishi, H. Toyoda, and H. Kugai, "Detection of fine iron particles in high speed scrolled wire by high Tc SQUID," IECE Trans. Electron., vol. E80-C, pp. 1247-1251, 1997.

[3] Y. Tavrin, M. Siegel, and J. H. Hinken, "Standard method for detection of magnetic defects in aircraft engine discs using a HTS SQUID gradiometer," IEEE Trans. Appl. Supercond., vol. 9, pp. 3809-3812, 1999.

[4] D. F. He and M. Yoshizawa, "Metal detector based on high-Tc rf SQUID," Physica C, vol. 378-381, pp. 1404-1407, 2002.

[5] M. Bick, P. Sullivan, D. L. Tilbrook, J. Du, B. Thorn, R. Binks, C. Sharman, K. E. Leslie, A. Hinsch, K. Macrae, and C. P. Foley, "Metal in food detection: HTS SQUID versus conventional technology," in Proc. ISEC 2003 Conf., Sydney, Australia, Jul. 7-11, 2003, paper PTh06.

[6] S. Tanaka, M. Natsume, M. Uchida, N. Hotta, T. Matsuda, Z. A. Spanut, and Y. Hatsukade, "Measurement of metallic contaminants in food with a high-Tc SQUID," Supercond. Sci. Technol., vol. 17, pp. 620-623, 2004.

[7] Y. Zhang, J. Schubert, N. Wolters, M. Banzet, W. Zander, and H.-J. Krause, "Substrate resonator for HTS rf SQUID operation," Physica C, vol. 372-376, pp. 282-286, 2002. 